\title{
Aromatase Inhibitors Versus Cabergoline for the Prevention of Ovarian Hyperstimulation Syndrome: a Prospective, Randomized, Double-Blind Study \\ Original
} Article

\author{
Walid M. Ataalla ${ }^{a}$, Tarek Abd Elhamid
}

${ }^{a}$ Departments of Obstetrics \& Gynaecology, ${ }^{b}$ Histopathology, Faculty of Medicine, Tanta University, Egypt, Department, Tanta University, Egypt

\begin{abstract}
Objective: To evaluate the effect of the aromatase inhibitor letrozole in the prevention of ovarian hyperstimulation syndrome (OHSS) in patients at risk during controlled ovarian hyperstimul $(\mathrm{COH})$.

Design: This is a prospective, randomized, double-blind study on women at risk of the development of OHSS during $\mathrm{COH}$ using gonadotropin-releasing hormone $(\mathrm{GnRH})$ antagonist protocol

Patients and Methods: This research was conducted at the Center of Assisted Reproduction, Om El-kora Hospital, Tanta, Egypt. Sixty patients, who were considered at risk of the development of OHSS during COH were enrolled in this study. Ovarian stimulation was performed using $\mathrm{GnRH}$, whereas cabergoline $(0.25 \mathrm{mg})$ given twice daily or letrozole $(2.5 \mathrm{mg})$ twice daily from the day of oocyte retrieval for one week.

Results: In the cabergoline group, two cases (6.6\%) developed mild OHSS and one case (3.3\%) developed moderate OHSS versus 8 (26.6\%) mild OHSS and $6(20 \%)$ moderate OHSS in the letrozole group. On the other hand, there was no statistically significant difference in the number of cases that developed severe OHSS in both groups where only one case (3.3\%) developed severe OHSS in the cabergoline group vs. two (6.6\%) cases in the letrozole group.

Conclusion: Although letrozle administration during the luteal phase of ART cycles can reduce the high E2 level in hyper-responding patients, but it cannot prevent the development of early OHSS. On the contrary, Cabergoline can effectively prevent the development of early OHSS.
\end{abstract}

Submitted: 25 August 2016, Accepted: 1 February 2017.

Key Words: Aromatase Inhibitors, Cabergoline, OHSS

Corresponding Author: Walid Attalla., February 2017, Vol. 7, No. 1., Tel.: 01223488672 E-mail: wma646@hotmail.com

ISSN: 2090-7265, February 2017, Vol. 7, No. 1

\section{INTRODUCTION}

Prevention of OHSS can be either primary or secondary prevention. Primary prevention means tailoring IVF treatment protocols according to the patient's primary risk factors, while the secondary prevention means the methods used to prevent the progression to OHSS in patients already showed excessive response to ovarian stimulation ${ }^{1}$. Individualizing IVF protocol includes modification of the starting dose of FSH, use of antagonist protocol instead of long agonist protocol, lowering the dose of hCG needed to trigger ovulation ${ }^{18}$ or using GnRHa for triggering ovulation in $\mathrm{GnRH}$ antagonist treated cycles $^{19}$. Secondary preventive measures include coasting ${ }^{18}$, withholding hCG and cycle cancellation $^{20}$, cryopreservation of all embryos ${ }^{21}$, intravenous administration of albumin ${ }^{22}$ or hydroxyethyl starch (HES) ${ }^{23}$ during or immediately after oocyte retrieval, use of cabergoline ${ }^{24}$ or the vasopressin V1a receptor antagonist, relcovaptan ${ }^{25}$.
Cabergoline, a dopamine antagonist prevents the VEGF effect on vascular system, i.e. reducing VEGF receptor 2 expression/ phosphorylation by a D2 receptor-mediated mechanism ${ }^{24,26}$. Cochrane Review by Tang et al. showed that cabergoline is effective in significantly reducing the incidence of moderate but not severe OHSS without significant effect on clinical pregnancy or miscarriage rates ${ }^{27}$. More recently, a systemic review by Leitao at el. showed that cabergoline is effective in preventing the occurance of both moderate and severe OHSS again without affecting clinical pregnancy rate ${ }^{28}$.

Though the role of elevated E2 in OHSS has not been confirmed, estradiol concentrations on the day of hCG administration seem to play a role in OHSS development ${ }^{29}$ and lowering E2 level helps to prevent $\mathrm{OHSS}^{30}$. One way to decrease estradiol is aromatase 
inhibitors and so this could be used to prevent OHSS when used in the early luteal phase by acting on the sustained corpora lutea.

Two randomized studies assessed the effect of letrozol adminstratuin in the luteal phase of IVF cycles in oocyte donors and both of them confirmed that letrozole reduces the luteal E2 levels in stimulated cycles $^{31,32}$.

\section{PATIENTS AND METHODS}

This prospective, randomized study was carried out in the Center of Assisted Reproduction, Om El kora Hospital, Tanta, Egypt. From January 2014 to December 2014, a total of 60 patients, who were considered at risk of the development of OHSS during $\mathrm{COH}$ were recruited. Informed consents obtained from all patients.

This study approved by the Clinical Research Ethics Committee.

\section{Inclusion criteria}

PCO patients and patients with ultrasonic picture of PCO, who became at risk of developing OHSSduring controlled ovarian hyperstimulation using antagonist protocol. OHSS defined by serum E2 level > 3000 $\mathrm{pg} / \mathrm{ml}$ and/or the development of $\geq 20$ follicles larger than $12 \mathrm{~mm}$ in diameter on the day of HCG.

We included only patients who agreed to cryopreserve all embryos to avoid late OHSS and to avoid the possibility of lower pregnancy rates secondary to letrozole mediated lowering of oestradiol levels.

\section{Exclusion criteria}

Patients, with E2 levels of 3000- $3500 \mathrm{pg} / \mathrm{ml}$, who refused to cryopreserve all embryos. Since our patients were given GnRH agoinst for final oocyte maturation that could lower the risk of OHSS itself, patients with E levels $>3500 \mathrm{Pg} / \mathrm{ml}$ were excluded.

All patients with E2 levels between 3000- 3500 $\mathrm{pg} / \mathrm{ml}$, who agreed to cryopreserve all embryos, were given HCG $5000 \mathrm{IU}$ for triggering ovulation thereafter randomized by lottery into two equal groups. Group I (30 patients) received cabergoline $0.25 \mathrm{mg}$ twice daily. Group II (30 patients) received letrozole $2.5 \mathrm{mg}$ twice daily from the day of oocyte retrieval and for one week. Randomization was done with both the physicians and the patients blinded.

After oocyte retrieval patients were instructed to report any of the following symptoms; lower abdominal discomfort, nausea, vomiting, diarrhea, bloating, abdominal distention, passing very small amounts of urine, swollen and tender leg or respiratory difficulty. Otherwise, ultrasound was performed for all patients one week after oocyte retrieval to measure ovarian size and for detection of ascitis.

\section{IVF protocol:}

All patients received antagonist stimulation protocol. All patients in each group received 150 IU of recombinant FSH, Gonal-F (Serono, Switzerland) daily for 4 days starting from the second day of menstruation. The dose of gonadotropins was adjusted according to the ovarian response on transvaginal ultrasound and serum oestradiol concentration starting from day 5 of stimulation. The GnRH antagonist cetrorelix $0.25 \mathrm{mg}$ (Cetrotide, Serono Laboratories, Aubonne, Switzerland) was started in all patients on a fixed day which is day 5 of stimulation and continued until day of HCG administration. I.M. injection of 5000 IU of HCG (Choriomon, IBSA, Switzerland) or subcutaneous injection of $0.2 \mathrm{mg}$ triptorelin (Decapeptyl; Ferring, $\mathrm{GmbH}$, Kiel, Germany) were administered when at least three follicles reached $\geq 17 \mathrm{~mm}$ in diameter. Ultrasound guided transvaginal oocyte retrieval was performed 3436- hours later. All embryos were frozen in order to decrease the risk of late OHSS.

\section{Hormone assay}

In the cycle preceding the stimulation cycle, $\mathrm{AMH}$ $(\mathrm{ng} / \mathrm{ml})$ was measured on any day of the cycle. Serum E2 $(\mathrm{pg} / \mathrm{ml}), \mathrm{LH}(\mathrm{iu} / \mathrm{l})$ and progesterone $(\mathrm{ng} / \mathrm{ml})$ were measured on the second day of menstruation of the stimulation cycle before starting gonadotropins. E2 was then measured during the stimulation cycle to assist monitoring of the ovarian response. Serum E2 level was compared in the two groups before stimulation, at day of hCG and 6 days after initiation of cabergoline or letrozole.

The development of mild, moderate or severe OHSS was compared in both groups.

\section{RESULTS}

This study included 60 patients. Patients were randomly distributed with 30 patients in each arm. Demographic data of the study patients are listed in Table 2. There were no significant differences in the patient characteristics in the two groups.

The ovarian responsiveness and treatment outcomes are compared in Table 3. No significant differences obtained between the two groups in E2 measurements before stating ovarian stimulation, on day 6 of stimulation or on the day of HCG. But a statistically significant 
different revealed on day 7 after oocyte retrieval where E2 level was significantly lower in the letrozole group compared to the cabergoline group.

Regarding OHSS, two cases $(6.6 \%)$ developed mild OHSS and one case $(3.3 \%)$ developed moderate OHSS in the cabergoline group. This was significantly lower than the number of cases that developed mild and moderate OHSS in the letrozole group which were $8(26.6 \%)$ and 6 $(20 \%)$ cases, respectively. On the contrary, no statistically significant difference obtained in cases that developed severe OHSS in both groups where only one case $(3.3 \%)$ developed severe OHSS in the cabergoline group compared to two $(6.6 \%)$ cases in the letrozole group.

Table 1: Patients' characteristics.

\begin{tabular}{lccc}
\hline & $\begin{array}{c}\text { cabergoline group } \\
\mathbf{n}=\mathbf{3 0})\end{array}$ & $\begin{array}{c}\text { letrozole group } \\
(\mathbf{n}=\mathbf{3 0})\end{array}$ & P value \\
\hline Age (Y) & $23.43 \pm 3.115$ & $24.53 \pm 2.897$ & 0.162 \\
\hline BMI (kg/m2) & $21.93 \pm 6.726$ & $23.70 \pm 2.667$ & 0.186 \\
\hline Basal FSH (IU/L) & $4.12 \pm 0.941$ & $3.87 \pm 1.008$ & 0.325 \\
\hline Basal LH (IU/L) & $5.46 \pm 1.339$ & $5.49 \pm 1.166$ & 0.927 \\
\hline AMH (ng/ml) & $5.05 \pm 1.245$ & $5.37 \pm 1.266$ & 0.338 \\
\hline Duration of infertility (Y) & $3.09 \pm 1.435$ & $3.67 \pm 1.155$ & 0.09 \\
\hline Type of infertility & & & 0.759 \\
\hline Primary & 23() & $24(\%)$ & 0.759 \\
\hline Secondary & $7(\%)$ & $6(\%)$ & 0.791 \\
\hline Main causes of infertility & $11(\%)$ & $10(\%)$ & 0.798 \\
\hline Tubal disease & $13(\%)$ & $12(\%)$ & 0.549 \\
\hline Male factor & $6(\%)$ & $8(\%)$ & \\
\hline Unexplained & & & \\
\hline
\end{tabular}

"significant $P<0.05$

Table 2: Criteria of stimulation cycles.

\begin{tabular}{lccccc}
\hline & $\begin{array}{c}\text { Cabergoline group } \\
(\mathbf{n}=\mathbf{3 0})\end{array}$ & Mean $\pm \mathbf{S D}$ & $\begin{array}{c}\text { Letrozole group } \\
(\mathbf{n}=\mathbf{3 0})\end{array}$ & Mean \pm SD & P value \\
\hline Days of stimulation & $(9.0-11.0)$ & $10 \pm 0.830$ & $(8.0-11.0)$ & $9.63 \pm 0.928$ & 0.112 \\
\hline Number of 75 IU FSH ampoules & $(16-25.0)$ & $18.97 \pm 2.833$ & $(15.0-23.0)$ & $18.7 \pm 2.667$ & 0.711 \\
\hline E2 $\mathbf{p g} / \mathbf{m l})$ & & & & \\
\hline E2, prestimulation & $(13.6-44.6)$ & $28.207 \pm 8.780$ & $(12.9-42.4)$ & $28.110 \pm 8.688$ & 0.966 \\
\hline E2, at day 6 of stimulation & $(1440.6-1811.2)$ & $1699.7 \pm 100.08$ & $(1569.6-1992.7)$ & $1732.8 \pm 111.43$ & 0.230 \\
\hline E2, at day of HCG & $(3035.5-3480.7)$ & $3221.9 \pm 137.93$ & $(3096.8-3469.1)$ & $3277.0 \pm 107.65$ & 0.086 \\
\hline E2, 7 day after oocyte retreival & $(1466.2-1650.8)$ & $1558.024 \pm 59.24$ & $(750.4-1050.9)$ & $905.043 \pm 104.86$ & $0.000^{*}$ \\
\hline No. of follicles on day of HCG & $(24-28)$ & $25.93 \pm 1.28$ & $(25-30)$ & $26.57 \pm 1.35$ & 0.068 \\
\hline No. of oocytes per retrieval & $(18-25)$ & $20.6 \pm 2.027$ & $(20-25)$ & $21.3 \pm 2.351$ & 0.222 \\
\hline OHSS & & & & & $0.038^{*}$ \\
\hline Mild & $2(6.6 \%)$ & & $6(26.6 \%)$ & & $0.045^{*}$ \\
\hline Moderate & $1(3.3 \%)$ & $2(6.6 \%)$ & & \\
\hline Severe & $1(3.3 \%)$ & & & & \\
\hline
\end{tabular}

"significant $P<0.05$ 


\section{DISCUSSION}

HCG for final oocyte maturation is well documented as the triggering factor for cascade of events, which results in the development of OHSS. These events were almost always accompanied by elevated serum E2 levels. Estradiol concentrations on the day of hCG administration seem to play a role in OHSS development ${ }^{29}$. Rapid rise of E2 together with other factors as large number of developing follicles on the day of hCG administration ( $>14$ follicles with a diameter of $11 \mathrm{~mm}$ ), and a large number of oocytes retrieved have been proposed as risk factors for developing $\mathrm{OHSS}^{17}$.

Coasting is a strategy to prevent OHSS by withholding gonadotrophins when a certain E2 level and/or a critical number of follicles are reached. When the E2 drops to a "safe" level, hCG is administered for final oocyte maturation. It is generally employed for a period less than 3 days ${ }^{18}$. It has been shown that levels of VEGF which is one of the vasoactive amines that play a role in the development of OHSS are reduced along with falling E2 levels during Coasting ${ }^{33}$.

In this study, we used the aromatase inhibitor letrozole which blocks the aromatization of androstenedione to estrogen to reduce estradiol concentrations and may help in preventing early OHSS. Letrozole was used from the day of oocyte retrieval and not during the follicular phase to avoid any deleterious effect on folliculogenesis or oocyte maturation through lowering of estradiol or accumulation of androgens.

Two randomized studies assessed the effect of letrozol administration in the luteal phase of IVF cycles in oocyte donors. Both confirmed that letrozole reduces the luteal E2 levels in stimulated cycles ${ }^{31,32}$ a prospective randomized controlled pilot study. Six oocyte donors were randomized after $\mathrm{COH}$ and successful egg retrieval to receive either $2.5 \mathrm{mg}$ of letrozole or a placebo. Donors were tested for serum estradiol, progesterone and LH on the day of hCG administration and day 4, 7 and 10 after oocyte retrieval. In the second study, twenty oocyte donors were randomized after $\mathrm{COH}$ and successful egg retrieval to receive either $2.5 \mathrm{mg}$ of letrozole or a placebo (folic acid tablets) for 5 days from the day of ovum pick-up. Donors were tested for serum estradiol, progesterone and LH the day of hCG administration and day 4, 7 and 10. Both studies showed significantly reduced serum E2 levels at 4, 7 and 10 days after oocyte retrieval compared to controls. The first study showed no difference in LH levels among treatment and control groups while the second study showed that letrozole appeared to significantly increased LH levels on days 7 and 10 after retrieval.
Similar to our study, Fatemi et al, used letrozole in the early luteal phase for prevention of OHSS in a 21-yearold patient which was at high risk for the development of OHSS during COH. On Day 9 of stimulation, E2 level was $5000 \mathrm{pg} / \mathrm{ml}$ and 50 follicles were visualized. Noteworthy, HCG 5000 IU was used for final oocyte maturation because of limited experience of the physician on duty. The patient was given letrozol $2.5 \mathrm{mg}$ twice daily and cabergoline 0.5 daily directly post oocyte retrieval up to the day of embryo transfer. 52 oocytes were retrieved of which 38 were Metaphase II. 23 oocytes were fertilized with ICSI, and on day three 13 embryos were biopsied for PGD. On day 5 after oocyte retrieval, one genetically normal embryo was transferred and at 7 weeks of gestation, a viable singleton pregnancy could be visualized on ultrasound. The patients did not develop any early nor late $\mathrm{OHSS}^{34}$.

Wang et al. conducted a study on 281 patients who were potential candidates for developing OHSS after $\mathrm{COH}$ in ART cycles using long GnRH agonist protocol. All participating patients met at least one of the following criteria: (1) Number of retrieved oocytes $\geq 20$; (2) mean number of follicles with a diameter greater than $14 \mathrm{~mm}$ was $\geq 20$; (3) serum E2 concentrations reached $\geq 8000$ $\mathrm{pg} / \mathrm{ml}$; (4) on the day of oocyte retrieval, the ovarian diameter was $>10 \mathrm{~cm}$; and $\backslash$ presentation of obviously symptoms of OHSS on the day of aspiration. Patients were given 6000-8000 IU of hCG for final oocyte maturation. The couples were counseled about the high risk of OHSS and all agreed to cancel fresh embryo transfer. Patients were divided into a treatment group $(\mathrm{n}=161)$ and control group $(\mathrm{n}=120)$. The patients in the treatment group were informed of treatment options: first group: received letrozole tab $2.5 \mathrm{mg}$ twice daily for 5 consecutive days, second group: received mifepristone tab $25 \mathrm{mg}$ twice daily for 3 consecutive days. Third group: received subcutaneous cetrotide $0.25 \mathrm{mg}$ daily for 5 consecutive days. Fourth group: received the three drugs together at the same time. The control group: received no special medication. All patients started the medication on the day after oocyte retrieval. There was significantly lower E2 level on days 2, 5 and 8 after oocyte retrieval in the letrozole and three-drug combination therapy group than in the other three groups. There was no significant difference in serum LH concentration on the same days and progesterone concentration on day 8 after oocyte retreival among the five groups $\mathrm{s}^{35}$.

A possible harmful effect of letrozole on oocyte maturation and embryo quality was revealed in previous studies. In context, Ehsan, et al. conducted a comparative study on one hundred and twenty patients who were potential candidates for developing OHSS after $\mathrm{COH}$ in assisted reproduction technology (ART) cycles using long GnRH agonist protocol. They compared the use of letrozole versus coasting in prevention of OHSS. Women 
at risk for OHSS "i.e., with serum E2 $>1634 \mathrm{pg} / \mathrm{ml}$ on day 8 of stimulation" were randomized to receive letrozole $(2.5$ $\mathrm{mg} /$ day if E2 level was $\geq 3000 \mathrm{pg} / \mathrm{ml}$ or every other day if E2 was $<3000 \mathrm{pg} / \mathrm{ml}$ starting from day 8 of stimulation till the day before hCG) or to undergo coasting. Though number of metaphase II oocyte retrieved was similar in both groups, number of grade I egg and of grade I embryo was lower in letrozole group compared to coasting group $^{36}$. Similarly, administration of AI in late follicular phase resulted in significantly lower number of metaphase II oocyte in stimulated rhesus monkey ${ }^{37}$.

On the other hand, It was observed that $>50 \%$ drop of E2 from initial level does not compromise the oocyte quality and implantation but prevent $\mathrm{OHSS}^{33}$. Moreover, oestradiol depletion in the vicinity of the oocyte did not impair its development, fertilization, development into morula, blastocyst and hatching blastocysts ${ }^{37-38}$. Additionally, the extremely high levels of E2 have a deleterious effect on gamete quality and/or uterine receptivity ${ }^{39,40}$.

To avoid late OHSS and also to avoid any harmful effect of letrozole on the endometrium secondary to lowering of estradiol that could be reflected on implantation and pregnancy rate, we included only patients who agreed to cryopreserve all embryos.

In ART cycles, rapid changes in the hormonal levels occur during the luteal phase. Just before oocyte aspiration, supraphysiologic E2 concentrations are reached, which is followed by a dramatic fall in E2 after oocyte aspiration. There is a further fall in steroidal levels by day 9 of oocyte trigger as the trophic effect of HCG on the corpus luteum gets weaned off ${ }^{41}$. The impact of $\% \mathrm{E} 2$ fall in the early and midluteal phase on pregnancy outcome during $\mathrm{COH}$ is controversial.

Stewart $e t a l^{42}$ were the first to identify a significant difference in serum E2 concentrations between conception and nonconception cycles in fertile women undergoing donor insemination.

Using the long down-regulation protocol, Sharara et $a l^{43}$ measured the ratio of $\mathrm{E} 2$ on day of $\mathrm{HCG}$ to midluteal E2 and found that sharp decline in E2 resulted in a significantly lower implantation and pregnancy rates. This raised the speculation that the E2 fall would compromise the peri-implantation endometrial development.

Alike, Levi et al. used the same protocol and found that the sharper the drop in E2 levels, the lower was the possibility of successful IVF outcome ${ }^{44}$. It was speculated that insufficient exposure of the endometrium to E2 might lower or inhibit maintenance of the effects of some factors like leukemia inhibiting factor, interleukin-1, TNF- $\alpha$, and interferone.
Also, the research by Ganesh et $a l^{45}$ revealed that E2 on day 7 after ET was significantly higher in the conception group as compared to the non pregnant group.

On the other hand, Friedler et al ${ }^{46}$ studied the luteal phase E2 levels and found no difference in the mean midluteal E2 levels and \% of E2 decline in the conception versus non conception cycles.

Moreover, one meta-analysis compared the effect of adding E2 to progesterone verses progesterone alone for luteal phase support in GnRH agonist as well as antagonist cycles. It was concluded that addition of E2 to progesterone does not increase the pregnancy rate ${ }^{47}$.

So, despite the decline of E2 after oocyte retrieval, there is certain E2 level above which pregnancy rate could be maintained. Thus, we need to know whether letrozole use during the luteal phase causes additional decline in E2 that may compromise endometrial development and pregnancy rate.

Our results showed that the incidence of mild and moderate OHSS was significantly lower with cabergoline compared to letrozole. But, no significant difference was detected in the incidence of severe OHSS between the two groups.

In a case report, Fatemi et al tested the coadministration of letrozole and cabergoline in the early luteal phase for prevention of OHSS in a high risk patient undergoing ovarian stimulation for IVF. Neither early nor late OHSS was developed. On day 5 after oocyte retrieval, one genetically normal embryo was transferred. By the $7^{\text {th }}$ week of gestation, a viable singleton pregnancy was visualized by ultrasound ${ }^{34}$.

In another trial, Wang et al. compared the use of letrozole, mifepristone, cetrotide and the three-drug combinations versus no use in the prevention of severe OHSS. No significant decrease in the incidence of severe OHSS in each of the four groups was obtained ${ }^{35}$.

Interestingly, Ehsan et al compared the use of letrozole versus coasting in the prevention of OHSS, whereas no severe OHSS developed and only $5 \%$ of cases developed mild to moderate OHSS in the letrozole group. On the other hand, in coasting group 3.3\% of patients developed severe OHSS and $8.3 \%$ of cases developed mild to moderate OHSS. But the difference between the two groups in the incidence of mild and moderate OHSS did not reach statistical significance. Also, no significant difference in pregnancy rate was obtained between the two groups ${ }^{36}$. 


\section{CONCLUSIONS}

Letrozle administration during the luteal phase of ART cycles can reduce the high E2 level in hyper-responding patients, but it cannot prevent the development of early OHSS in these patients On the contrary, cabergoline can effectively prevent the development of early OHSS.

\section{CONFLICT OF INTERESTS}

There are no conflict of interest.

\section{REFERENCES}

1. Humaidan P, Quartarolo J, Papanikolaou EG. Preventing ovarian hyperstimulation syndrome: Guidance for the clinician. Fertil Steril 2010; 94(2): $389-400$

2. Tan BK, Mathur R. Management of ovarian hyperstimulation syndrome. Produced on behalf of the BFS policy and practice committee. Hum Fertil 2013; 16(3): 151-159.

3. Goldsman MP, Pedram A, Dominguez CE, Ciuffardi I, Levin E, Asch RH. Increased capillary permeability induced by human follicular fluid: A hypothesis for an ovarian origin of the hyperstimulation syndrome. Fertil Steril 1995; 63(2): 268-272.

4. Tollan A, Holst N, Forsdahl F, Fadnes HO, Oian P, Maltau JM. Transcapillary fluid dynamics during ovarian stimulation for in vitro fertilization. Am J Obstet Gynecol 1990; 162(2): 554-558.

5. Aboulghar MA, Mansour RT. Ovarian hyperstimulation syndrome: Classifications and critical analysis of preventive measures. Human Reproduction Update 2003; 9(3): 275-289.

6. Mathur RS, Akande AV, Keay SD, Hunt LP, Jenkins JM. Distinction between early and late ovarian hyperstimulation syndrome. Fertil Steril 2000; 73(5): 901-907.

7. Mathur R, Evbuomwan I, Jenkins J. Prevention and management of ovarian hyperstimulation syndrome. Curr Obstet Gynaecol 2005; 15(2): 132-138.

8. Soares SR, G'omez R, Sim'on C, Garc'iaVelasco JA, Pellicer A. Targeting the vascular endothelial growth factor system to prevent ovarian hyperstimulation syndrome. Hum Reprod Update 2008; 14(2): 321-333.

9. Wang TH, Horng SG, Chang CL, Wu HM, Tsai YJ, Wang HS, et al. Human chorionic gonadotropininduced ovarian hyperstimulation syndrome is associated with up-regulation of vascular endothelial growth factor. J Clin Endocrinol Metab 2002; 87(7): 3300-3308.

10. Naredi N, Talwar P, Sandeep K. VEGF antagonist for the prevention of ovarian hyperstimulation syndrome: Current status. Med J Armed Forces India 2014; 70(1): 58-63.

11. Kasum M. New insights in mechanisms for development of ovarian hyperstimulation syndrome. Coll Antropol 2010; 34(3): 1139-1143.

12. Joint Society of Obstetricians and Gynaecologists of Canada-Canadian Fertility Andrology Society Clinical Practice Guidelines Committee; Reproductive Endocrinology and Infertility Committee of the SOGC; Executive and Council of the Society of Obstetricians; Gynaecologists of Canada; Board of the Canadian Fertility and Andrology Society, Shmorgun D, Claman P. The diagnosis and management of ovarian hyperstimulation syndrome. J Obstet Gynaecol Can 2011;33(11): 1156- 1162.

13. Lee TH, Liu CH, Huang CC, Wu YL, Shih YT, Ho HN, et al. Serum anti-Mullerian hormone and estradiol levels as predictors of ovarian hyperstimulation syndrome in assisted reproduction technology cycles. Hum Reprod 2008; 23(1): 160-167.

14. Delvigne A, Rozenberg S. Epidemiology and prevention of Ovarian Hyper Stimulation Syndrome (OHSS): A review. Hum Reprod Update 2002; 8(6): 559-577.

15. Papanikolaou EG, Humaidan P, Polyzos NP, Tarlatzis B. Identification of the high-risk patient for ovarian hyperstimulation syndrome. Semin Reprod Med. 2010 Nov; 28(6): 458 -462.

16. Practice Committee of American Society for Reproductive Medicine. Ovarian hyperstimulation syndrome. Fertil Steril 2008; 90 (5 suppl): S188-S193.

17. Papanikolaou EG, Pozzobon C, Kolibianakis EM, Camus M, Tournaye H, Fatemi HM, et al. Incidence and prediction of ovarian hyperstimulation syndrome in women undergoing gonadotropinreleasing hormone antagonist in vitro fertilization cycles. Fertil Steril 2006; 85(1): 112-120.

18. Mathur RS, Tan BK. British Fertility Society Policy and Practice Committee: Prevention of ovarian hyperstimulation syndrome. Hum Fertil 2014; 17(4): 257-268.

19. Kol S, Mannaerts M, Itskovitz-Eldor. Triggering of preovulatory LH surge after treatment with the 
GnRH antagosint in IVF cycles and the prevention of ovarian hyperstimulation syndrome (OHSS). J Soc Gynecol Invest 2000; 94 (7): 300A.

20. Mathur R, Sumaya W. Prevention and management of ovarian hyperstimulation syndrome. Obstet Gynaecol Reprod Med 2008; 18(1): 18-22.

21. Angelo AD. Ovarian hyperstimulation syndrome prevention strategies: Cryopreservation of all embryos. Semin Reprod Med 2010; 28(6): 513-518.

22. Navot D, Bergh PA, Laufer N. Ovarian hyperstimulation syndrome in novel reproductive technologies: Prevention and treatment. Fertil Steril 1992; 58(2): 249- 261.

23. Youssef MA, Al-Inany HG, Evers JL, Aboulghar M. Intra-venous fluids for the prevention of severe ovarian hyperstimulation syndrome. Cochrane Database of Syst Rev 2011; (2): Article ID CD001302

24. Garcia-Velasco JA. How to avoid ovarian hyperstimulation syndrome: A new indication for dopamine agonists. Reprod Biomed Online 2009; 18(Suppl 2): S71-S75.

25. Cenksoy C, Cenksoy PO, Erdem O, Sancak B, Gursoy R. A potential novel strategy, inhibition of vasopressin-induced VEGF secretion by relcovaptan, for decreasing the incidence of ovarian hyperstimulation syndrome in the hyperstimulated rat model. Eur J Obstet Gynecol Reprod Biol 2014; 174(1): 86-90.

26. Basu S, Nagy JA, Pal S, Vasile E, Eckelhoefer IA, Bliss VS, et al. The neurotransmitter dopamine inhibits angiogenesis induced by vascular permeability factor/vascular endothelial growth factor. Nat Med 2001; 7(5): 569-574.

27. Tang $\mathrm{H}$, Hunter $\mathrm{T}$, Hu $\mathrm{Y}$, Zhai SD, Sheng $\mathrm{X}$, Hart RJ. Cabergoline for preventing ovarian hyperstimulation syndrome. Cochrane Database Syst Rev 2012; (2): Article ID CD008605.

28. Leitao VM, Moroni RM, Seko LM, Nastri CO, Martins WP . Cabergoline for the prevention of ovarian hyperstimulation syndrome: Systematic review and meta-analysis of randomized controlled trials. Fertil Steril 2014; 101(3): 664-675.

29. Verwoerd GR, Mathews T, Brinsden PR. Optimal follicle and oocyte numbers for cryopreservation of all embryos in IVF cycles at risk of OHSS. Reprod Biomed Online 2008; 17(3): 312 -317.
30. Sher G, Zouves C, Feinman M, Maassarani G. 'Prolonged coasting': An effective method for preventing severe ovarian hyperstimulation syndrome in patients undergoing in-vitro fertilization. Hum Reprod 1995; 10(12): 3107- 3109.

31. Fatemi HM, Popovic-Todorovic B, Donoso P, Papanikolaou E, Smitz J, Devroey P. Luteal phase oestradiol suppression by letrozole: A pilot study in oocyte donors. Reprod Biomed Online 2008; 17(3):307 -311.

32. Garcia-Velasco JA, Quea G, Piro M, Mayoral M, Ruiz M, Toribio M, et al. Letrozole administration during the luteal phase after ovarian stimulation impacts corpus luteum function: A randomized, placebocontrolled trial. Fertil Steril 2009; 92:222 -225.

33. García-Velasco JA, Isaza V, Quea G, Pellicer A. Coasting for the prevention of ovarian hyperstimulation syndrome: much ado about nothing?. Fertil Steril 2006; 85(3): 547 -554.

34. Fatemi HM, Kyrou D, Papanikolaou EG, Al Buarki H, Garcia Velasco J. Letrozole and Cabergoline Co-administration in the Early Luteal Phase for Prevention of OHSS in a High Risk Patient Undergoing Ovarian Stimulation for IVF. J Fert In Vitro 2012; 2(4): Article ID 1000111.

35. Wang YQ, Luo J, Xu WM, Xie QZ, Yan WJ, Wu GX, et al. Can steroidal ovarian suppression during the luteal phase after oocyte retrieval reduce the risk of severe OHSS? J Ovarian Res. 2015; 8: 63.

36. Ehsan M, Begum MS, Beby HA, Siddiqui M, Begum MR. Aromatase Inhibitor for Prevention of Ovarian Hyperstimulation Syndrome (OHSS). Androl Gynecol: Curr Res 2014; 2:3. Article ID 1000125.

37. Zelinski-Wooten MB, Hess DL, Baughman WL, Molskness TA, Wolf DP, et al. Administration of an aromatase inhibitor during the late follicular phase of gonadotropin treated cycles in rhesus monkeys: effects on follicle development, oocyte maturation and subsequent luteal function. J Clin Endocrinol Metab 1993; 76(4): 988- 995.

38. Weston AM, Zelinski-Wooten MB, Hutchison JS, Stouffer RL, Wolf DP. Development potential of embryos produced by in-vitro fertilization from gonadotropin-releasing hormone antagonist-treated macaques stimulated with recombinant follicle stimulating hormone alone or in combination with luteinizing hormone. Hum Reprod 1996; 11(3): 608- 613 . 
39. Simón C, Garcia Velasco JJ, Valbuena D, Peinado JA, Moreno $\mathrm{C}$, et al. Increasing uterine receptivity by decreasing estradiol levels during the preimplantation period in high responders with the use of a follicle-stimulating hormone step-down regimen. Fertil Steril 1998; 70(2): 234- 239.

40. Valbuena D, Martin J, de Pablo JL, Remohí J, Pellicer A, Simón C. Increasing levels of estradiol are deleterious to embryonic implantation because they directly affect the embryo. Fertil Steril 2001; 76(5): 962- 968.

41. Smitz J, Erard P, Camus M, Devroey P, Tournaye H, Wisanto A, et al. Pituitary gonadotrophin secretory capacity during the luteal phase in superovulation using GnRH-agonists and HMG in a desensitization or flare-up protocol. Hum Reprod. 1992; 7(9): $1225-1229$.

42. Stewart DR, Overstreet JW, Nakjama ST, Lasley BL. "Enhanced ovarian steroid secretion before implantation in early human pregnancy" J Clin Endocrinol Metab. 1993; 76:1470-1476.

43. Sharara FI, McClamrock HD. Ratio of estradiol concentration on the day of human chronic gonadotropin administration to mid-luteal oestradiol concentration is predictive of in-vitro fertilization outcome. Hum Reprod 199914(11): 2777-2782.

44. Levi R, Ozyurek SE, Ege N, Goker T, Tavmergen EH. Early and mid-luteal phase blood estradiol levels after ovum pick-up and pregnancy rates after ICSI Cycles. J Reprod Med 2004; 49(2):108-114.

45. Ganesh A, Goswami S, Chattopadhyay R, Chakraborty C, Chaudhury K, Chakravarty BN. Luteal phase estradiol level: A potential predictive marker for successful pregnancy in invitro fertilization/ intracytoplasmic sperm injection. Fertil Steril. 2009; 91(4):1018-1022.

46. 46.Friedler S, Zimerman A, Schachter M, Raziel A, Strassburger D, Ron El R. The midluteal decline in serum estradiol levels is drastic but not deleterious for implantation after in vitro fertilization and embryo transfer in patients with normal or high responses. Fertil Steril 2005; 83(1): 54-60.

47. 47. Jee BC, Suh CS, Kim SH, Kim YB, Moon SY. Effect of estradiol supplementation during the luteal phase of in vitro fertilization cycles: A meta-analysis. Fertil Steril 2010;93(2): 428- 436. 